\title{
Überwachung von Fettsäuren in Biogasprozessen - Neue Entwicklungen für die Online-Messung
}

\author{
J. Zosel', M. Schelter ${ }^{1}$, M. Schlegel ${ }^{2}$, J. Kretzschmar ${ }^{3}$, K. Ostermann ${ }^{4}$, G. Rödel' ${ }^{4}$ K. Hahne ${ }^{4}$, \\ J. Posseckardt ${ }^{1}$, C. Schirmer ${ }^{1}$, M. Mertig ${ }^{1,4}$ \\ ${ }^{1}$ Kurt-Schwabe-Institut für Mess- und Sensortechnik e.V. Meinsberg, \\ Kurt-Schwabe-Straße 4, 04736 Waldheim, Deutschland \\ 2Universität Rostock, Professur für Agrartechnologie und Verfahrenstechnik, \\ Justus-von-Liebig Weg 6b, 18059 Rostock, Deutschland \\ ${ }^{3}$ DBFZ Deutsches Biomasseforschungszentrum gemeinnützige $\mathrm{GmbH}$, \\ Torgauer Str. 116, 04347 Leipzig, Deutschland \\ ${ }^{4}$ Technische Universität Dresden, 01062 Dresden, Deutschland \\ zosel@ksi-meinsberg.de
}

\section{Zusammenfassung}

Flüchtige Fettsäuren (engl. volatile fatty acids, VFA) wie Essig-, Propion-, Butter- und Valeriansäure sind wichtige Inhaltsstoffe für die Beurteilung der Stabilität von Biogasprozessen. Insbesondere bei der Verarbeitung organischer Abfälle unterschiedlicher Herkunft wie Speiseresten zu Biogas ist die zeitnahe Überwachung dieser Zwischenprodukte für die Prozesssicherheit entscheidend. Eine weitere Motivation für das Online-Monitoring von VFA ergibt sich aus der Zielstellung, Biogasprozesse so zu flexibilisieren, dass mit den entsprechenden Anlagen auch größere Bedarfsschwankungen im Stromnetz berücksichtigt werden können. Bislang werden die VFA in Biogasprozessen als Summenparameter beispielsweise durch eine Bestimmung des Verhältnisses aller VFA zum gesamten anorganischen Kohlenstoff (FOS/TAC) oder sporadisch durch Laboranalysen an manuell entnommenen Proben gemessen. Diese Vorgehensweise beinhaltet neben einem erheblichen Zeitverzug auch eine Reihe von Fehlerquellen, die die Zuverlässigkeit dieser Offline-Messungen signifikant einschränken. Es besteht deshalb der dringende Wunsch, für die Weiterentwicklung von Biogasprozessen zuverlässige Sensoren oder Messsysteme zu schaffen, mit denen die VFA online ohne größeren Zeitverzug zuverlässig in einem engen Zeitraster überwacht werden können.

Im Beitrag werden übersichtsweise zwei innovative erfolgversprechende Ansätze zur Lösung dieser Messaufgabe vorgestellt, bei denen einerseits neue, mit elektroaktiven Biofilmen bedeckte Elektroden sowie andererseits lebende Hefezellen zum Einsatz kommen, die optisch auf eindringende Analyte reagieren. Außerdem wird ein in der Praxis bereits erfolgreich getestetes automatisches Messsystem für VFA vorgestellt, das die quasi-kontinuierliche und hochselektive Messung von VFA in einem kontinuierlichen Strom von Biogas-Kondensat ermöglicht. Ein breiter Messbereich von 0,015 bis $20 \mathrm{~g} / \mathrm{L}$ (Essigsäure) und eine relativ lange Betriebszeit ohne Wartung (>1 Monat) sind charakteristische Merkmale dieses neu entwickelten Messsystems.

Keywords: VFA-Messung,Biogas,Biofilm-Sensor,Hefezelle,Online-Messung

\section{Einleitung}

Die Umwandlung organischer Stoffe zu Biogas durch Gärprozesse erfolgt in mehreren Stufen über eine Reihe von Zwischenprodukten [1]. Für die Charakterisierung der Kinetik der einzelnen Stufen, die dynamische Prozessregelung und die Gewährleistung der Prozesssicherheit ist eine genaue Kenntnis der Konzentrationen der Zwischenprodukte im Fermenter, ihrer räumlichen Verteilung und ihrer zeitlichen Entwicklung notwendig [2]. Insbesondere der Einfluss flüchtiger Fettsäuren (VFA) wie Essig-, Propion-, Butter- und Valeriansäure ist in den letzten Jahren intensiv untersucht worden [3]. Im Resultat dieser Arbeiten gehören die Konzentrationen dieser Intermediate zu den wichtigsten Indikatoren für die Stabilität von Biogasprozessen [4]. Vor allem bei der Abfallvergärung und beim dynamischen Betrieb von Biogasanlagen für die flexible $\mathrm{Be}$ - 
reitstellung von Elektroenergie ist das kontinuierliche prozessnahe Monitoring von VFA zur Gewährleistung der Prozessstabilität erforderlich [5]. Bislang erfolgt die Beobachtung dieser Konzentrationen in Biogasprozessen nahezu ausschließlich durch die externe Offline-Analyse von manuell entnommenen Proben. Neben hohen Kosten ist diese Vorgehensweise mit weiteren Nachteilen verknüpft:

- relativ große Messfehler durch Probenahme und Probentransport,

- geringe zeitliche Dichte der Messwerte und

- hoher Zeitversatz zwischen Probenahme und Messergebnis.

Da mit dieser Methode bislang ein zeitnahes zuverlässiges Prozessmonitoring von VFA nicht möglich war, wurden verschiedene Entwicklungsansätze verfolgt, die ein Online-Monitoring ermöglichen könnten bzw. deren technische Eignung bereits nachgewiesen wurde [612]. Aus dem Stand der Forschung und Entwicklung lässt sich jedoch auch schlussfolgern, dass zuverlässige und kostengünstige Methoden und Geräte für die VFA-Bestimmung in Biogasmedien, die sich ohne höheren Aufwand auch an bestehende Biogasanlagen adaptieren lassen, weiterhin eine aktuelle Zielstellung für die Branche darstellen. Die in diesem Beitrag beschriebenen Arbeiten sind auf diese Zielstellung gerichtet. Die verschiedenen dargestellten Grundansätze werden im folgenden Abschnitt beschrieben. Sie bestehen darin,

1. die Reaktionen unterschiedlicher biologischer Systeme wie Bakterien und Hefezellen auf VFA elektrisch bzw. optisch zu erfassen sowie

2. die VFA automatisch, langzeitstabil und hochselektiv mit einem feldtauglichen GCMesssystem online zu bestimmen.

\section{Messmethoden}

\section{A) Biologische Sensorik}

Bei dem in Abb.1 gezeigten bioelektrochemischen Sensor werden elektroaktive Bakterien wie Geobacter sp. als Rezeptoren verwendet. Diese Bakterien sind in der Lage, im Rahmen ihres Energiestoffwechsels organische Substanzen wie Acetat vollständig $\mathrm{zu} \mathrm{CO}_{2} \mathrm{zu}$ oxidieren und polarisierte Elektroden als terminalen Elektronenakzeptor ihrer Atmungskette zu nutzen („Elektrodenatmung“) [13]. Solche elektroaktiven Bakterien bilden selbstständig Biofilme aus und übertragen Elektronen u.a. direkt durch membrangebundene Cytochrome oder sogenannte "nanowires“ auf die mit ca. 0,4 V gegen Standardwasserstoff-Elektrode polarisierten Elektroden. Die Acetatkonzentration wird auf diese Weise als Stromfluss erfasst.

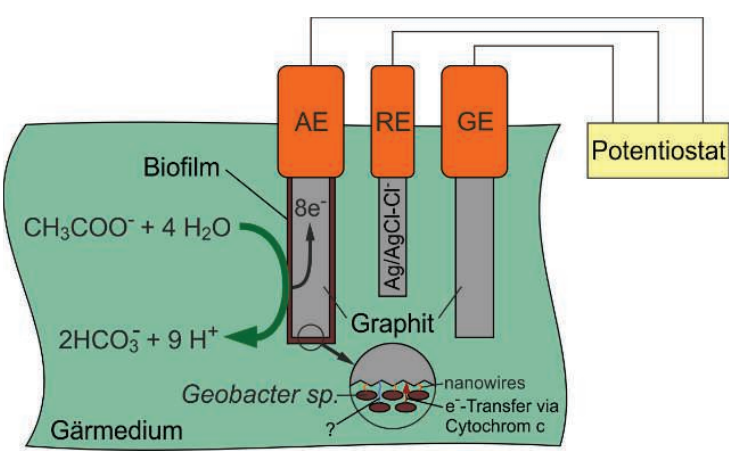

Abb. 1: Bioelektrochemischer Sensor unter Nutzung von Biofilmen aus Bakterien der Gattung Geobacter sp. (?: Der Elektronentransfer im Biofilm wird gegenwärtig intensiv erforscht.)

Eine weitere Möglichkeit stellt die in den letzten Jahren intensiv untersuchte gentechnische Modifizierung von Hefezellen dar, durch die dann verschiedene Analyte in der Umgebung dieser Zellen detektierbar sind. Der Nachweis erfolgt durch Aufnahme dieser Analyte in die Zellen, wie in Abb. 2 skizziert, und die selektive Exprimierung bestimmter Gene, die wenige Stunden nach Erstkontakt eine von außen optisch messbare Fluoreszenz auslösen.

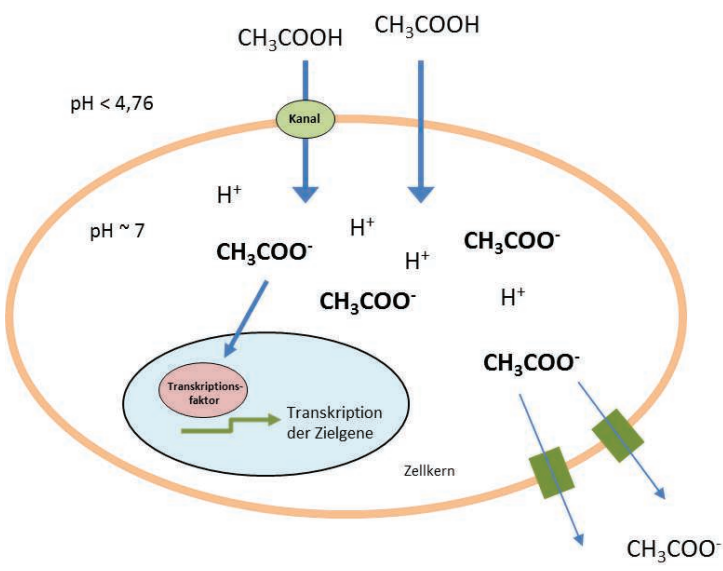

Abb. 2: Schematische Darstellung der Aufnahme und Metabolisierung von Essigsäure durch eine Hefezelle (modifiziert nach [14])

Zur Herstellung solcher Hefezellen für die Detektion von Essigsäure wurde die Promotorsequenz (genetischer "Schalter"), an die ein Essigsäure-spezifischer Transkriptionsfaktor bindet und damit den Promotor/"Schalter" aktiviert, mit einem Gen für ein rot-fluoreszierendes Protein verknüpft. Die Einbringung des entsprechenden genetischen Konstruktes in Hefezellen ist im Beitrag von Tietze et al. in diesem Tagungsband genauer beschrieben. 
Da es sich bei diesen Sensorhefen um genmodifizierte Organismen handelt, müssen diese für die Feldmessung so in einer Messzelle gehalten werden, dass sie in Kontakt mit dem Messmedium kommen, jedoch nicht frei in die Umgebung austreten können. Eine solche Messzelle, die auch die optische Auswertung der Fluoreszenz der Zellen ermöglicht, ist im Beitrag von Schirmer et al. in diesem Tagungsband beschrieben.

Zur Beurteilung der Vitalität der Hefezellen ist eine Messmethode entwickelt worden, die auf der Impedanzspektroskopie beruht. Nähere Angaben zu dieser Methode finden sich im Beitrag von Posseckardt et al. in diesem Tagungsband.

Gegenüber herkömmlichen Analysemethoden bietet die Messung von VFA mit Bakterien bzw. zellulären Systemen den entscheidenden Vorteil, dass hier nur der bioverfügbare Anteil der VFA im Gärmedium gemessen wird [15].

\section{B) Automatisches Messsystem}

Das Schema des automatischen Messsystems für VFA aus Biogasprozessen ist in Abb. 3 dargestellt. Das Grundkonzept beruht auf der Erkenntnis, dass sich die VFA-Konzentrationen im Gärmedium weitgehend im Biogas-Kondensat abbilden [16]. Das in einem integrierten Kühler gesammelte Biogaskondensat wird mit einer neu entwickelten Injektionseinheit in ein feldtaugliches GC-Messsystem eingespritzt. Das gesamte System wird über eine neuartige, speicherprogrammierbare Steuerung (SPS) geführt. Die eingespritzte Analytmenge lässt sich an die gefundenen VFA-Konzentrationen anpassen, so dass Auflösung und maximal messbare Konzentrationen dynamisch optimierbar sind. Die Einspritzung erfolgt in einen Split-Injektor, der etwa 1/30 des Analytes auf die FFAP-Trennsäule führt.

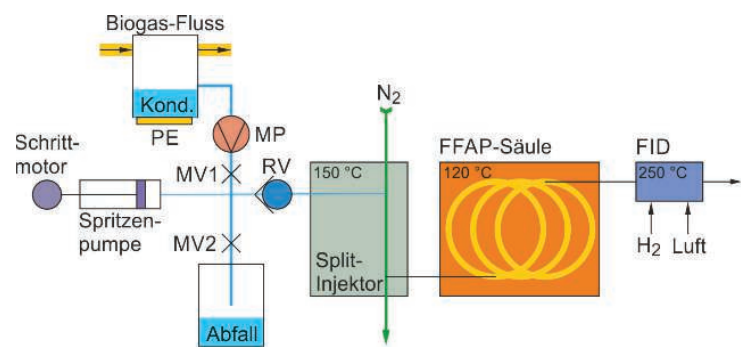

Abb. 3: Schema des automatisierten OnlineMesssystems zur VFA-Bestimmung in Biogasprozessen:

MP Membranpumpe, MV1/2 Magnetventile, PE Peltierelement,

Kond. Kondensat-Kühler, RV Rückschlagventil, FID Flammenionisationsdetektor, FFAP Polyethylenglykol-2-nitroterephthalsäureester

\section{Ergebnisse}

\section{A) Biologische Sensorik}

Für den in Abb. 1 gezeigten bioelektrochemischen Sensor wurden bereits der Messbereich, die Ansprechzeiten sowie Querempfindlichkeiten gegenüber Propionat und Butyrat untersucht $[17,18]$. Wie in Abb. 4 gezeigt, lässt sich Acetat unter Laborbedingungen zwischen 0,5 und $4 \mathrm{mmol} \cdot \mathrm{L}^{-1}$ in Echtzeit bestimmen [17]. Die Querempfindlichkeiten gegenüber Butyrat und Propionat sind minimal und äußern sich in Form eines additiven Basisliniensignals (Abb. 4 und [18]). Versuche in Fermentern unter realen Prozessbedingungen zeigen einen linearen Zusammenhang zwischen Stromsignal und Acetatkonzentration [19]. Zum Schutz des Rezeptors vor toxischen Substanzen und zur Vermeidung von Wechselwirkungen mit anderen Mikroorganismen ist dessen Abschirmung mit einer Membran vorgesehen $[20,21]$. Zur Überwachung des Sensors in situ ist die elektrochemische Impedanzspektroskopie vorgesehen. Erste umfassende Untersuchungen mit dieser Methode zeigen, dass es schwierig ist, zwischen einer Biofilminaktivierung und einer Konzentrationsänderung des Analyten (Acetat) zu differenzieren. EIS-Messungen beim Ruhepotential und/oder bei sehr niedrigen Frequenzen ermöglichen eventuell eine bessere Abgrenzung beider Vorgänge.

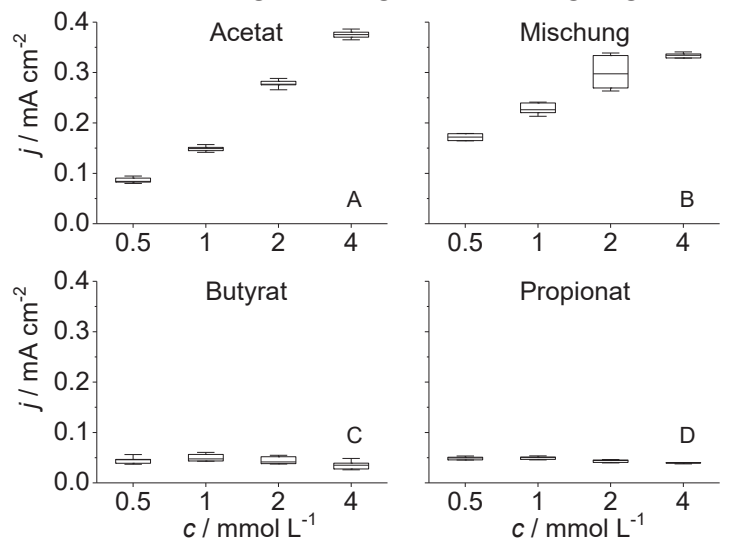

Abb. 4: Chronoamperometrisches Signal des in Abb. 1 gezeigten bioelektrochemischen Sensors, $E_{\text {Anode: }}$ 0,397 V vs. SHE in $50 \mathrm{mM}$ Phosphatpuffer; $A$ : Acetat; B: äquimolare Mischung aus Acetat, Propionat, und Butyrat, C: Butyrat und D: Propionat. Boxen: mittlere $50 \%$ der Messwerte; Whisker: Minimum und Maximum [18]

Die Reaktion genmodifizierter Hefezellen auf undissoziierte Essigsäure im Nährmedium durch Fluoreszenz ist in Abb. 5 dargestellt. Eine nicht dem Analyt ausgesetzte Kontrollgruppe zeigt kein Signal. 

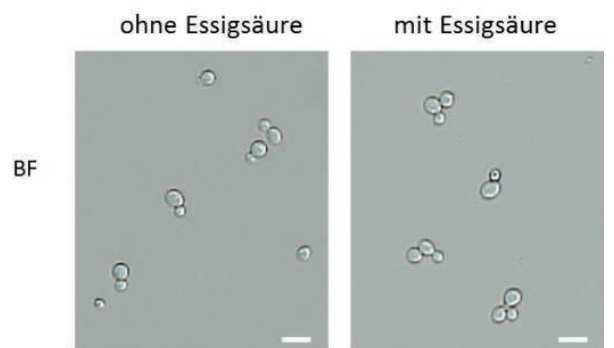

$\mathrm{FL}$
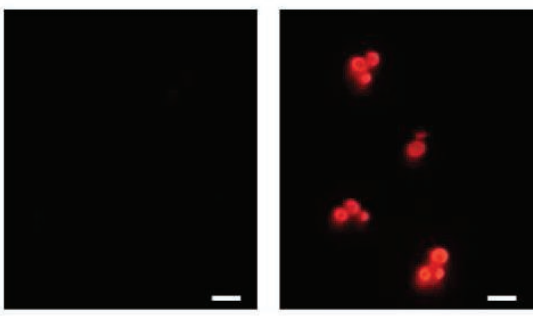

OL
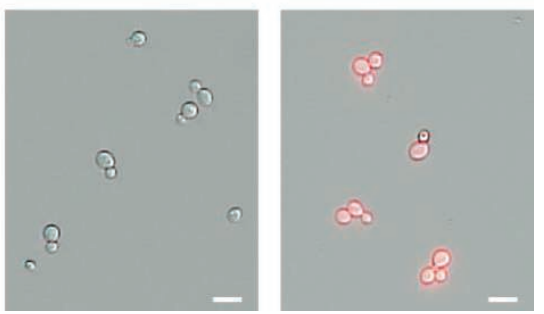

Abb. 5: Mikroskopische Aufnahmen der Sensorhefen in An-oder Abwesenheit von $15 \mathrm{mM}$ Essigsäure (BF: Hellfeld, FL: Fluoreszenz, OL: Überlagerung). Die weißen Balken entsprechen $10 \mu \mathrm{m}$. Die Mikroskopie erfolgte mit einem Keyence Biozero 8000 (Objektiv: Plan Apo 20x NA 0,75) bei 20-facher Vergrößerung.

Eine größere Anzahl dieser genmodifizierten Hefezellen, die in einer Durchflusszelle immobilisiert sind, reagieren in gleicher Weise auf den Kontakt mit Essigsäure, wie die entsprechende Kurve in Abb. 6 bestätigt. Die Zeitdauer zwischen Erstkontakt und Anstieg des Fluoreszenzsignals beträgt mehrere Stunden.

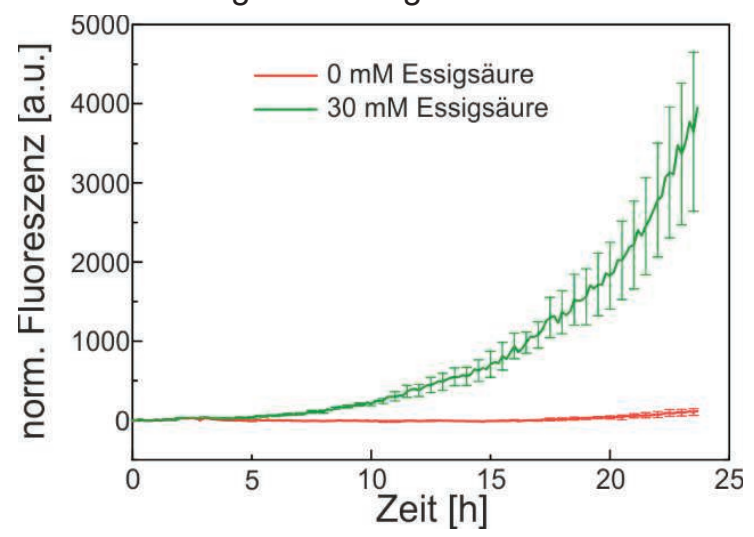

Abb. 6: Fluoreszenzsignal einer Vielzahl genmodifizierter Hefezellen bei Inkubation in Essigsäure-haltigem Nährmedium

\section{B) Automatisches Messsystem}

Eine Besonderheit des in Abb. 3 gezeigten automatischen Messsystems mit chromatographischer Trennung besteht in der im Split-Injektor fest installierten Einspritzkanüle. Dadurch wird der sonst beobachtete Verschleiß des Septums vermieden und die Einspritzposition variiert nicht. Am Austritt der Kanüle herrscht somit immer die Temperatur des Injektors, die hier auf $150{ }^{\circ} \mathrm{C}$ geregelt wird. Diese Injektion mit heißer Kanüle ermöglicht die Ausbildung kürzerer und höherer Peaks, wie die Chromatogramme in Abb. 7 belegen.

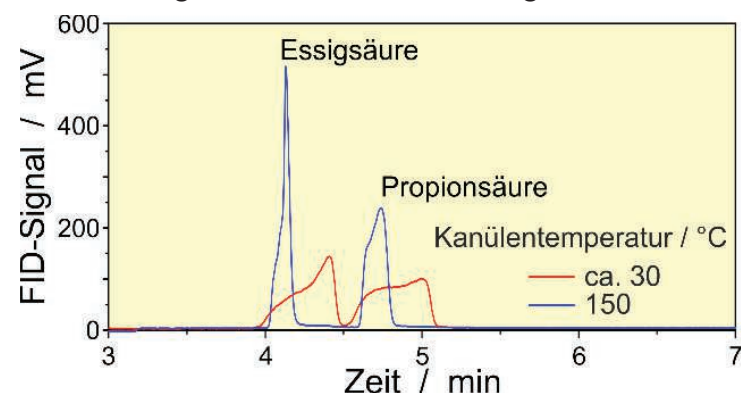

Abb. 7: Chromatogramme einer Kalibrierlösung mit der Essigsäurekonzentration 980 $\mathrm{mg} / \mathrm{L}$ und der Propionsäurekonzentration $540 \mathrm{mg} / \mathrm{L}$ bei Einspritzung mit kalter und heißer Kanüle, eingespritztes Volumen $=21 \mu \mathrm{L}$.

Im automatischen Betrieb wurden Standardlösungen injiziert und Chromatogramme aufgezeichnet, von denen Beispiele in Abb. 8 dargestellt sind. Die Resultate belegen, dass sich Peakhöhe und Peakfläche aller gemessenen VFA linear zueinander ändern. Die bei der Kalibrierung gewählten Konzentrationen bilden sich bei Propion-, Butter- und Valeriansäure nicht in dem Maß ab, wie es die Sensitivität des eingesetzten FID erwarten lässt, da es beim Einspritzen wahrscheinlich zu einem Siedeverzug kommt. Schwankungen von Peakhöhe und -fläche aller gemessenen VFA zwischen nacheinander aufgenommenen Chromato-grammen unterschreiten unter sonst gleichen Bedingungen die Grenze $\pm 12 \%$ vom Messwert und sind für den geplanten Einsatz des Messsystems ausreichend gering. Da diese Schwankungen der einzelnen VFA-Werte untereinander keine Korrelation aufweisen, wirken sich zufällige Fehler beim eingespritzten Volumen offenbar deutlich weniger aus, als die zufälligen Fehler bei der chromatographischen Trennung, Detektion und anschließenden Peakauswertung. Um eine möglichst lange Standzeit der verwendeten Kapillarsäule zu ermöglichen, wurde die Säulentemperatur im Messbetrieb konstant gehalten. 


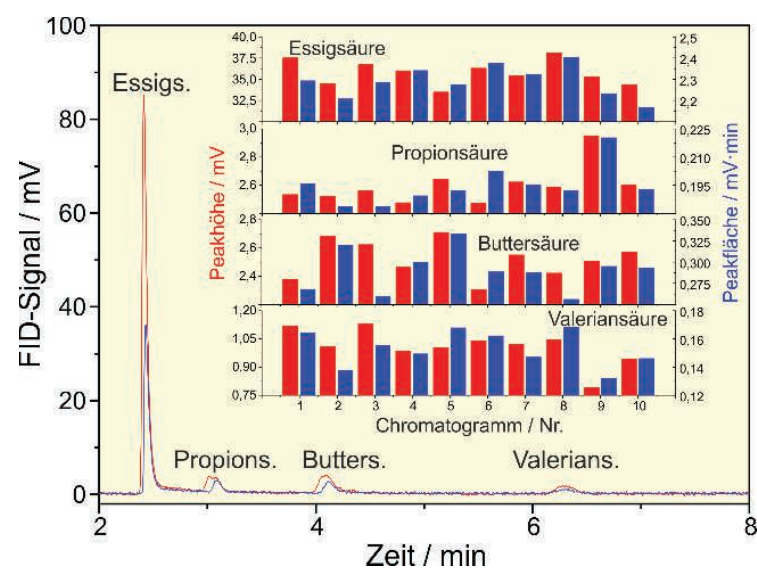

Abb. 8: Chromatogramme zweier Kalibrierlösungen mit den Essigsäurekonzentrationen 120 und $60 \mathrm{mg} / \mathrm{L}$, den Propionund Buttersäurekonzentrationen 6 und $3 \mathrm{mg} / \mathrm{L}$ und den Valeriansäurekonzentrationen 3 und 1,5 mg/L beim Einspritzvolumen $25 \mu \mathrm{L}$ und Peakparameter von 10 aufeinanderfolgenden Chromatogrammen der Kalibrierlösung mit 60 mg/L Essigsäure

Adsorptionszeiten und Peakbreiten nehmen wegen dieser isothermen Prozessführung mit zunehmender Retentionszeit erwartungsgemäß zu und die Sensitivität für die höheren VFA verringert sich geringfügig.

In Abb. 9 ist eine mit dem automatischen Messsystem aufgenommene Tagesverlaufskurve der Essigsäurekonzentration im Kondensat einer Biogas-Anlage dargestellt. Die Anlage wurde bei relativ niedrigen Raumbelastungen unter $1 \mathrm{~kg}$ OTS $/ \mathrm{m}^{3} \cdot \mathrm{d}$ betrieben, so dass im Gärmedium und im Biogas nur sehr geringe Essigsäurekonzentrationen zu erwarten waren. Das Ergebnis belegt, dass die untere Messgrenze des Systems den Nachweis im Kondensat auch bei dieser Prozessführung noch ermöglicht.

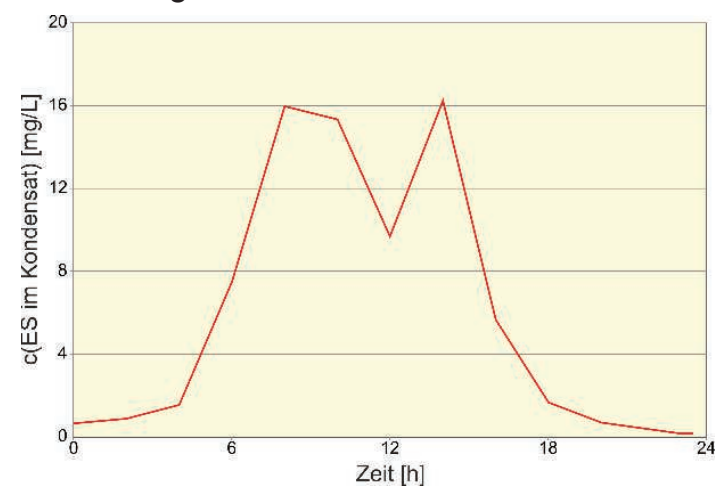

Abb. 9: Typische Tagesverlaufskurve der Essigsäurekonzentration im BiogasKondensat einer Anlage mit geringer Raumbelastung. (Mess. Peakfläche)

\section{Zusammenfassung}

Im Beitrag wurden verschiedene Ansätze vorgestellt, die auf die prozessnahe Messung flüchtiger Fettsäuren (VFA) bei der Biogasherstellung gerichtet sind und sich in ihren Messprinzipien als auch in ihren Parametern grundlegend unterscheiden. Der bioelektrochemische Sensor nutzt elektroaktive Bakterien und kann die biologisch verfügbaren Acetat-Ionen im Gärmedium direkt in den gewünschten Konzentrationsbereichen nachweisen. Für einen künftigen großtechnischen Einsatz dieses Sensors sind weitere Forschungsarbeiten notwendig, die auf die Erhöhung der mechanischen Stabilität des Biofilms und auf die sichere Beurteilung der Vitalität der Sensorbakterien gerichtet sind.

Das innovative Messverfahren zur Detektion von Essigsäure unter Nutzung genmodifizierter Hefezellen erfasst die nicht-dissoziierte Essigsäure und ist durch eine hohe Sensitivität und Selektivität gekennzeichnet. Im Vergleich zu den anderen Ansätzen weist es relativ lange Ansprechzeiten auf. Hier stehen weiterhin Fragestellungen im Fokus, die die Haltbarkeit der Sensorzellen sowie Inhaltsstoffe im Messmedium betreffen, die sich ungünstig auf die Vitalität der Zellen auswirken können. Auch dieses Verfahren bietet den Vorteil der selektiven Messung des biologisch verfügbaren Anteils von Essigsäure.

Das weiterhin vorgestellte feldtaugliche automatisierte Messsystem zur hochselektiven Bestimmung der wichtigsten VFA in Biogas-Kondensaten lässt sich mit überschaubarem Installationsaufwand an bestehende Biogasanlagen adaptieren. Der breite Messbereich von 0,015 bis $20 \mathrm{~g} / \mathrm{L}$ für Essigsäure und von 0,001 bis $10 \mathrm{~g} / \mathrm{L}$ für die weiteren VFA ermöglicht den Einsatz bei nahezu allen bislang bekannten Biogas-Applikationen. Erste Betriebserfahrungen zeigen, dass ein wartungsfreier Betrieb über mehrere Wochen möglich ist und das System über mehrere Monate im Dauerbetrieb eingesetzt werden kann.

\section{Danksagung}

Die Entwicklung von Ganzzellsensoren zur Messung von Inhaltsstoffen in Biogassubstraten wird vom Bundesministerium für Bildung und Forschung im Rahmen des Wachstumskerns BioSAM (Förderkennzeichen 03WKCL6) gefördert. Die den Arbeiten am automatischen Messsystem sowie am bioelektrochemischen Sensor zugrunde liegenden Vorhaben wurden mit Mitteln des Bundesministeriums für Wirtschaft und Energie (BMWi) gefördert. Die Verantwortung für den Inhalt dieser Arbeit liegt bei den Autoren, die für die Förderungen danken. 


\section{Literatur}

[1] D. Deublein, A. Steinhauser, Biogas from Waste and Renewable Resources. WileyVCH, Weinheim. (2008)

[2] H. Hahn, B. Krautkremer, K. Hartmann, M. Wachendorf, Renew. Sustain. Energy Rev. 29, 383-393 (2014); doi: 10.1016/j.rser.2013.08.085

[3] I.H. Franke-Whittle, A. Walter, C. Ebner, H. Insam, Waste Manag 34, 2080-2089 (2014); doi: 10.1016/j.wasman.2014.07.020

[4] K. Boe, J.P. Steyer, I. Angelidaki, Water Sci Technol. 57, 661-666 (2008); doi: 10.2166/wst.2008.046.

[5] E. Mauky, H.F. Jacobi, J. Liebetrau, M. Nelles, Bioresour. Technol. 178, 262-269 (2015); doi: 10.1016/j.biortech.2014.08.123

[6] N. Tippkötter, A. Deterding, R. Ulber, Engineering in Life Science 8, 62-67 (2008); doi: 10.1002/elsc.200820227

[7] P. F. Pind, I. Angelidaki, B. K. Ahring, Biotechnology and Bioengineering 82, 5461(2003); doi: 10.1002/bit.10537

[8] C. Wolf, D. Gaida, A. Stuhlsatz, S. McLoone, M. Bongards, Organic Acid Prediction in Biogas Plants Using UV/vis Spectroscopic Online-Measurements. In: K. Li et al. (Eds.) LSMS/ICSEE 2010, Part I; CCIS 97, pp. 200-206, Springer Verlag Berlin, Heidelberg (2010)

[9] A. Walte, W. Münchmeyer, B. Linke, I. Härtge, B. Becker, A. Wehrhan, A. Buchholz, Verwendung eines Propionsäure erkennenden Gas-SensorArrays zur Regelung von Vergärungsprozessen, Gülzower Fachgespräche Band 27, Messen, Steuern, Regeln bei der Biogaserzeugung. S. 72-83. (2007)

[10] H. Andree, H. F. Jacobi, E. Hartung, Online-NIRS-Messung an Biogasanlagen. Gülzower Fachgespräche Band 27, Messen, Steuern, Regeln bei der Biogaserzeugung, S. 84-99. (2007)

[11] M. Schelter, J. Zosel, W. Oelßner, M. Mertig, Verfahren und Vorrichtung zur Bestimmung flüchtiger organischer Säuren in Gasgemischen, Patentanmeldung DE1020130219434 (2013)

[12] E. Palacio-Barco E., F. Robert-Peillard, J.L. Boudenne, B. Coulomb, Analytica Chimica Acta 668, 74-79 (2010); doi: 10.1016/j.aca.2009.12.019

[13] U. Schroeder, Phys. Chem. Chem. Phys. 9, 2619-2629 (2007); doi: $10.1039 / 003627 m$
[14] S. Giannattasio, N. Guaragnella, M. Zdralević, E. Marra, Front. Microbio. 4:33 (2013); doi: 10.3389/fmicb.2013.00033

[15] S. Daunert, G. Barrett, J.S. Feliciano, R.S. Shetty, S. Shrestha, W. Smith-Spencer, Chem. Rev. 100, 2705-2738. (2000); DOI: $10.1021 / \mathrm{cr} 990115 \mathrm{p}$

[16] M. Schlegel, T. Geick, Überwachungseinrichtung für eine Biogasanlage. Patentanmeldung DE 10 2012112085 B4 (2017)

[17] J. Kretzschmar, L.F.M. Rosa, J. Zosel, M. Mertig, J. Liebetrau, F. Harnisch, Chem. Eng. Technol. 39, 637-642 (2016); doi: 10.1002/ceat.201500406

[18] J. Kretzschmar, C. Koch, J. Liebetrau, M. Mertig, F. Harnisch, Sens. Actuators B Chem. 241, 466-472 (2017); doi: 10.1016/j.snb.2016.10.097

[19] J. Kretzschmar, P. Böhme, J. Liebetrau, M. Mertig, F. Harnisch, Chem. Eng. Technol. (under review)

[20] D.R. Lovley, ISME J 11, 327-336 (2016); doi: 10.1038/ismej.2016.136

[21] A.-E. Rotaru, P.M. Shrestha, F. Liu, M. Shrestha, D. Shrestha, M. Embree, K. Zengler, C. Wardman, K.P. Nevin, D.R. Lovley, Energy Environ. Sci. 7, 408-415 (2014); doi: 10.1039/C3EE42189A 